\title{
Analog driver for synchronized resonant and quasistatic MOEMS mirrors
}

\author{
M. Lenzhofer ${ }^{1}$, A. Frank ${ }^{1}$, T. Sandner ${ }^{2}$, A. Kenda ${ }^{1}$, A. Tortschanoff ${ }^{1}$ \\ ${ }^{1}$ Carinthian Tech Research AG, Villach, Austria, \\ ${ }^{2}$ Fraunhofer Institute for Photonic Microsystems, Dresden, Germany
}

\begin{abstract}
Oscillating MOEMS mirrors achieve more and more interest in miniaturized applications like picoprojection devices, bar-code scanners ${ }^{1}$, spectrometers ${ }^{2}$, endoscopes, 3D distance scanners and others. To obtain better physical properties, especially an increased aperture in scanning devices, more mirrors have to be syncronized with each other. This implys a major challenge because the MOEMS elements show a spread in their mechanical proporties like resonance frequency and the response curve of the component. This paper shows two strategies to either syncronize resonant driven mirrors and on the other hand also presents the possibility to syncronize two quasitatic driven mirrors. Both driver circuits are built up in an analog manner to control the different types of mirrors. In this contribution we present the principle of the resonant driver concept with its drawbacks and also the principle of the quasistatic circuit, show the achieved experimental results with an approximately $470 \mathrm{~Hz}$ quaistatic mirror and demonstrate its capabilities but also show its limitations.
\end{abstract}

\section{INTRODUCTION}

A number of MOEMS based projection displays, imaging devices, barcode readers, and infrared imaging cameras have been developed for industrial, medical, and consumer market. Especially for scanner products one fast oscillating mirror axis or even two axes to draw a 2D raster pattern are necessary. To achieve a greater field of view an array of synchronized micromechanical devices can be used.

The resonant driving technology of scanning mirrors has several advantages, including high mirror deflection angles even at relatively low driving voltages and minimal energy consumption. Additionally they are shock and vibration resistant and feature high scanning rates caused by the low mass of the mirror element.

To guarantee proper functionality, meaning a stable resonant oscillation with well-defined amplitude even under varying environmental conditions it is essential to implement a closed loop control. This is done by integrating a PSD device to detect the position of the mirror element for both type of mirrors. Another advantage is that if more driver stages are supplied with the same input signal they are automatically synchronized because they lock onto the same reference signal.

In this contribution we present our driver circuits for a resonant driven and a quasistatic driven mirror device including the feedback scheme, and show first experimental results with a $23 \mathrm{kHz}$ resonant MOEMS mirror and a quasistatic mirror. While our circuits are specially designed and adjusted to control a special type of electrostatically driven MOEMS mirrors, the basic concept is viable for all kind of resonant and quasistatic micro actuators.

\section{MOEMS DEVICES AND DRIVING PRINCIPLES}

Since the motion of the MOEMS mirror is not linear but represents a quasi-harmonic function, it is necessary to measure the movement of the mirror. The accuracy evidently depends on the application. While for simple tasks just the fact that the mirror is oscillating might be sufficient, more sophisticated applications quite rapidly require high precision for example display applications.

\subsection{Resonant scanner mirror}

The resonant micromechanical mirrors used for our development were fabricated at the Fraunhofer IPMS using CMOS compatible technology ${ }^{3}$. Fig. 2. 1 shows a photograph of the used device. It consists of a plate suspended by two torsional springs and two driving comb-electrodes. Underneath the mirror plate and the torsional springs the bulk silicon is removed. 


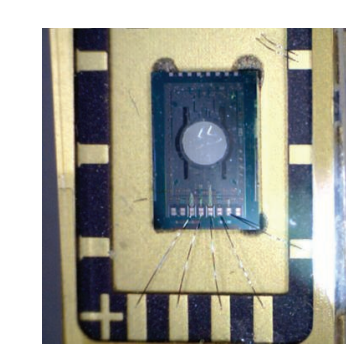

Fig. 1. Photograph of the resonant mirror used to test our driving concept.

Therefore, the deflection angle is not limited geometrically ${ }^{4}$. The vertical sides of the comb driving electrodes form with the mirror plate a variable capacitance, which value depends on the deflection angle. If a voltage is applied to the driving electrodes an electrostatic torque accelerates the plate towards its rest position. To prevent a deceleration of the movement after passing the rest position the voltage must be switched off. At the maximum deflection angle the voltage is switched on again. This driving principle is schematically illustrated in Fig. 2.
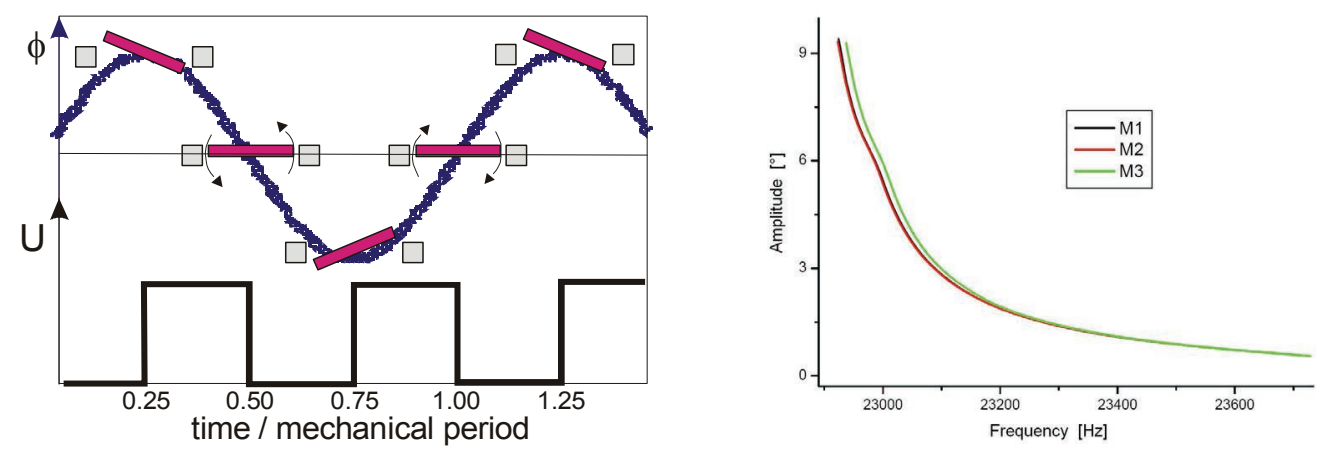

Fig. 2. Left: Sketch of the driving signal and the excitation waveform of the mirror plate. The voltage is switched off exactly at the cross over of the mirror plate oscillation and switched on at maximum deflection again, if the driving frequency exactly corresponds to twice the resonance frequency. Right: Mirror deflection angle dependent on the mirror frequency. The bandwidth where the main deflection is achieved is very small $<100 \mathrm{~Hz}$.

The resonance frequency of the MOEMS mirrors used in this work was around $23 \mathrm{kHz}$. The driving voltage to control the mirrors was in the range of $80 \mathrm{~V}$ up to a maximum of $120 \mathrm{~V}$. The mirrors are driven electrostatically with a pulsed driving voltage close to the double of their eigenfrequency. The amplitude is frequency dependent and maximal when being driven near the resonance frequency, Fig. 2. Mirror oscillation is initiated with a frequency sweep from higher to lower frequencies.

\subsection{Quasistatic mirror}

The quasistatic micromechanical mirrors used for our development are commercially available devices from Mirrorcle Technologies ${ }^{\circledR}$. Fig. 3 shows a photograph of the device we used. The MEMS actuator is fabricated in monolithic single crystal silicon and consists of 2-dimensional, gimbal-less vertical comb driven structures ${ }^{5}$. The mirror plate is fabricated in a separate SOI process and metalized before it is bonded to the actuator. The MOEMS mirror achieves a maximal amplitude of approximately $6.56^{\circ}$ at $123 \mathrm{~V}$. The resonant frequency depends on the used axis and is between $466 \mathrm{~Hz}$ and $470 \mathrm{~Hz}$.

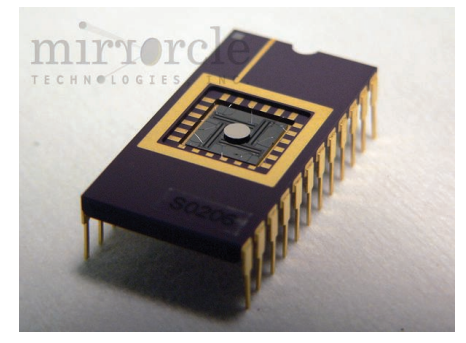

Fig. 3. Photograph of the quasistatic mirror used to test our driving concept.

To drive this MOEMS device a differential high voltage scheme with an additional bias voltage is used, which can be adjusted to prevent damage of the device. The advantage of this method is that it linearizes the device characteristic and provides smooth transitions when moving from one quadrant to the other. Fig. 4 shows the basic diagram of this driving method and the measurements done by mirrorcle. 


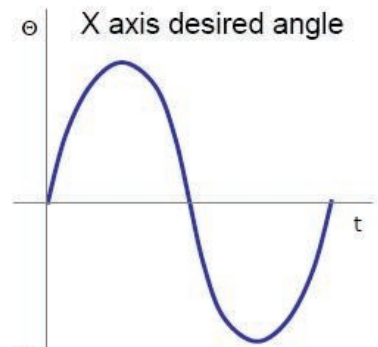

(a)

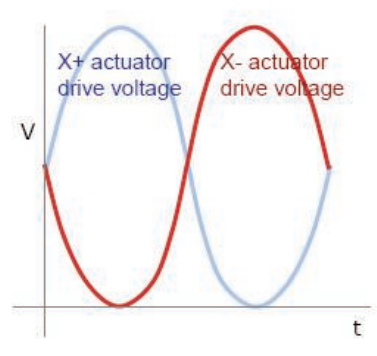

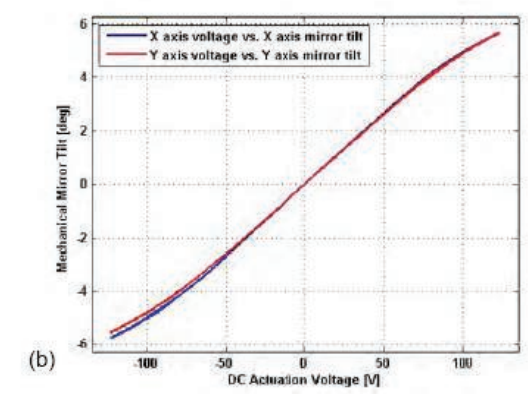

Fig. 4. a.) Deflection angle of the mirror plate with the linearized 4-quadrant driving scheme. b.) Mirror deflection angle dependent on the applied driving voltage.

\section{SYSTEM INTEGRATION AND RESULTS}

Fig. 5 shows the developed driver box that contains two independent high voltage drivers to syncronize two resonant and two quasistatic mirror devices. For the feedback path two dimensional PSD circuits are used, which generate an output voltage of $\pm 3 \mathrm{~V}$ depending on the power of the reference laser diode. Because of the fact that the implemented high voltage driver board accepts levels of approximately $\pm 9 \mathrm{~V}$ to achieve the maximal output voltage of up to $+140 \mathrm{~V}$, an additional power supply was inserted that supplys the output amplifiers. To control the resonant drivers a square waveform is applied to the Sub-D plug, which also varies in frequency to start up the mirror device. Also the set point signal for the PID controller is applied to the Sub-D plug. All other signals are just supply wires and indicator lines from the PSD device to achieve the possibility to monitor or adjust the optical configuration.

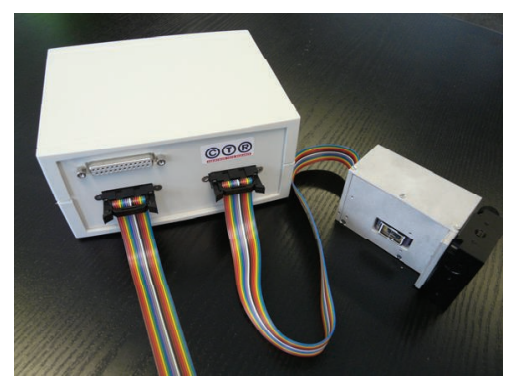

Fig. 5. Photograph of the developed high voltage driver box to drive two synchronized resonant or quasistatic mirrors.

\subsection{Phase-Locked-Loop driver for resonant mirror control}

The realized concept for driving the resonant mirror provides the integration of the MOEMS element into a phase-locked loop (PLL) circuit, where a voltage controlled oscillator can be automatically synchronized ("locked") to a periodic input signal $\mathrm{f}_{\mathrm{i}}$. The basic PLL circuit consists of three components connected in a feedback loop, shown in fig. 6. Namely a voltage controlled oscillator (VCO), a phase detector (PD) and a low-pass filter (LPF). The output frequency of the oscillator $f_{\text {osc }}$ is proportional to its input voltage, that is generated by the filtered output pulses $\mathrm{v}_{\mathrm{phi}}$ of the phase detector. The loop is locked onto the incoming signal $f_{i}$, if the oscillator signal is exactly equal to the periodic input signal.

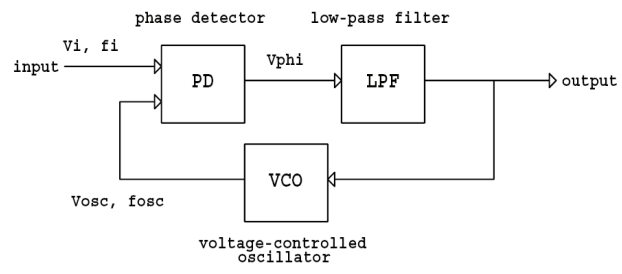

Fig. 6. Block diagram of the realized PLL loop to drive the resonant mirror.

The input driving signal $f_{i}$ is generated by an FPGA, while the feedback signal is generated by the PSD device, which measures the mirror movement. The output voltage of the VCO is adjusted to guarantee the maximal output voltage of the HV driver stage.

Another aspect is that the mirror device has to be driven with twice the mechanical frequency. In the feedback loop the PSD monitors the position signal, so that the controller has to generate a signal corresponding to the mechanical frequency. Thus the mirror acts as frequency divider in the closed loop. 


\subsection{Measurement results achieved with the resonant MOEMS mirror}

It was just quite a challange to drive the mirror in a controlled state. First of all the mirror requires a decreasing frequency sweep, which starts out of band and is then slowly adjusted towards its mechanical resonance frequency. This aspect demands that the PLL's free running frequency is set to the starting frequency of the mirror element. Fig. 7 shows the control principle of the circuit. The range of the input signal $f_{i}$ spans from $f_{\min }$ to $f_{\max }$ and is called lock range, where the PLL remains in the locked condition. If the PLL is initially locked and $f_{i}$ becomes smaller than $f_{\min }$, or exceeds $f_{\max }$, the circuit fails to keep $f_{\text {osc }}$ equal to $f_{i}$ and so the PLL becomes unlocked resulting in an oscillation at the center or free-running frequency $f_{0}$. The lock state can be established again, if the incomming signal $f_{i}$ gets close enough to $f_{o}$ again. This range between $\mathrm{f}_{\mathrm{i}}=\mathrm{f}_{\mathrm{O}}-\mathrm{f}_{\mathrm{c}}$ and $\mathrm{f}_{\mathrm{i}}=\mathrm{f}_{\mathrm{o}}+\mathrm{f}_{\mathrm{c}}$ is called capture range and is smaller than the lock range.

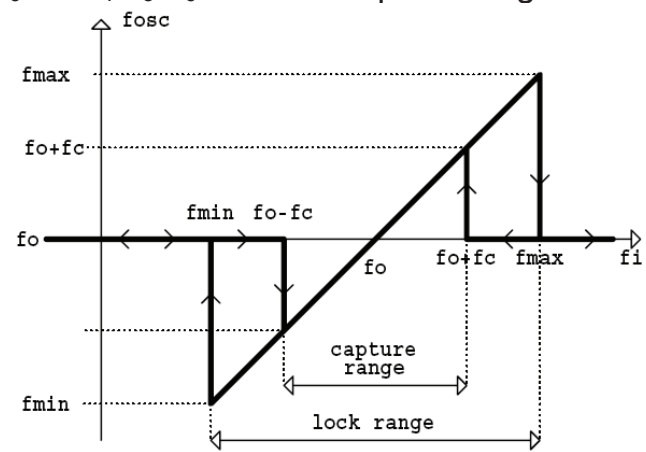

Fig. 7. Steady state $f_{\text {osc }}\left(f_{i}\right)$ characteristic of the PLL circuit.

The practical tests showed that in a certain range the circuit worked quite well, but if the frequency was slowly adjusted towards the mechanical resonance frequency of the mirror the concept failed. It was not possible to adjust the frequency settings of the PLL circuit in a way that the PLL remained locked closer than $80 \mathrm{~Hz}$ to its mechanical frequency. This sounds quite good, but the last few $\mathrm{Hz}$ cause the main deflection angle, see Fig. 2, since the coherency is not linear. The reason for this phenomena is that there is a small phase delay between the mirror position signal and the input signal $\mathrm{f}_{\mathrm{i}}$. If in the first moment the mirror actuates too slow, the error signal of the PD adjusts the control voltage of the oscillator leading to a higher frequency, causing to be too high for the next controller adjustment. This means that a small phase jitter or oscillation is generated that is too high for an accurate driving concept.

\subsection{PID driver circuit for quasistatic mirror control}

To control the quasistatic axis an analog PID controller was designed. Fig. 8 shows the realized circuit. It consists of an first order input filter followed by an input amplifer, which can be very flexible configured, either to use it as inverting or non inverting amlifier or impedance amplifier. This flexibility was necessary to achieve the possibility to use different laser modules, which lead to higher or lower signals on the PSD. The next stage is an adder circuit that substracts the actual value from the desired set point value. Afterwards the difference is weighted by three independent stages representing the integral, the diffrential and the proportional value of the PID controller. The feature to adjust all the values independently is very important, because every MOEMS miror has sligtly different parameters. The transfer function of the implemented controller is given by:

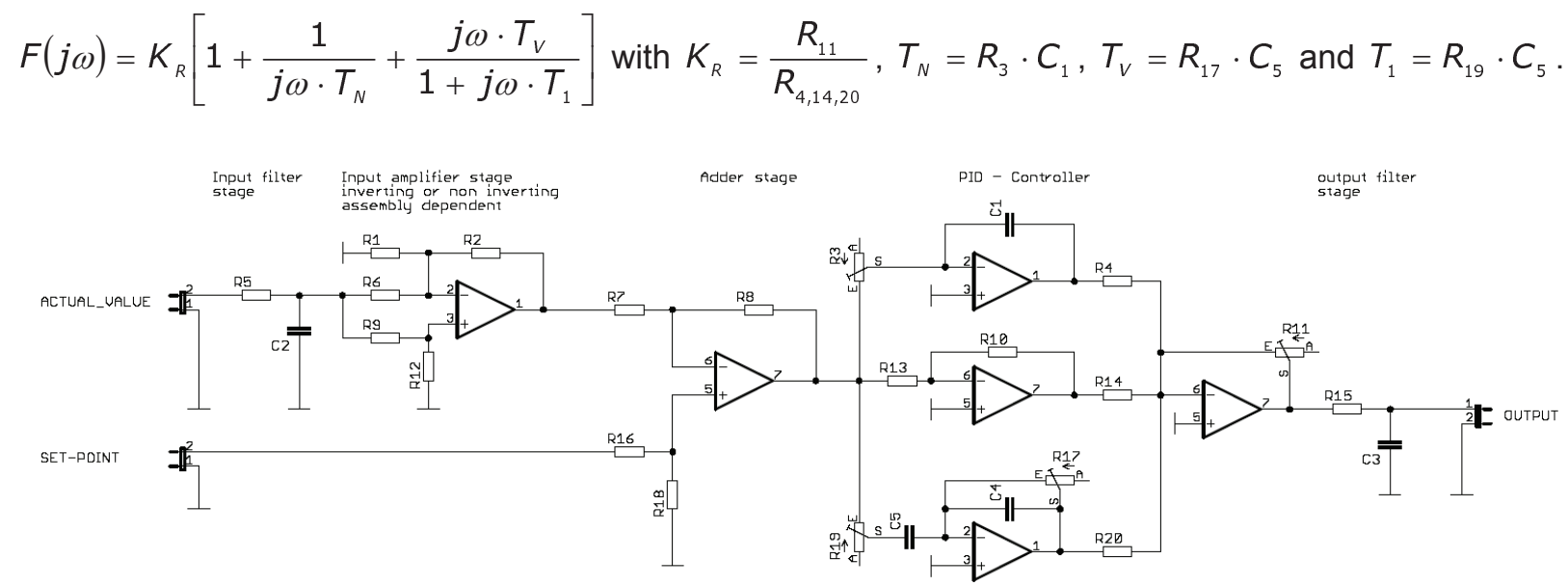

Fig. 8. Schematic of the realized PID control loop. 


\subsection{Measurement results achieved with the quasistatic MOEMS mirror}

Before starting the adjustment of the PID controller first of all the step response signal of the quasistatic mirror was measuered. Fig. 9 shows the measured signals.
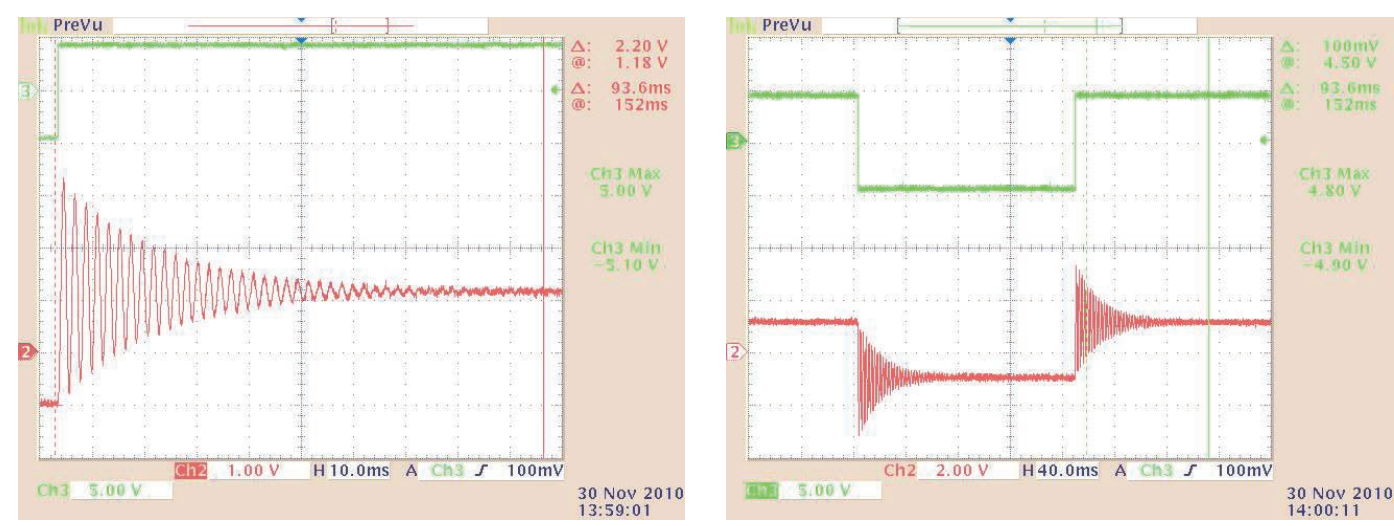

Fig. 9. Left: Detailed zoom of the step response signal. The mirror oscillates with its eigenfrequency for about $80 \mathrm{~ms}$ before reaching a quasi stationary position. Right: The mirror response is identical on both, raising and falling slope of the control signal.

In the next step the PID controller parameters were adjusted, so that the following signals could be measured, fig. 10.
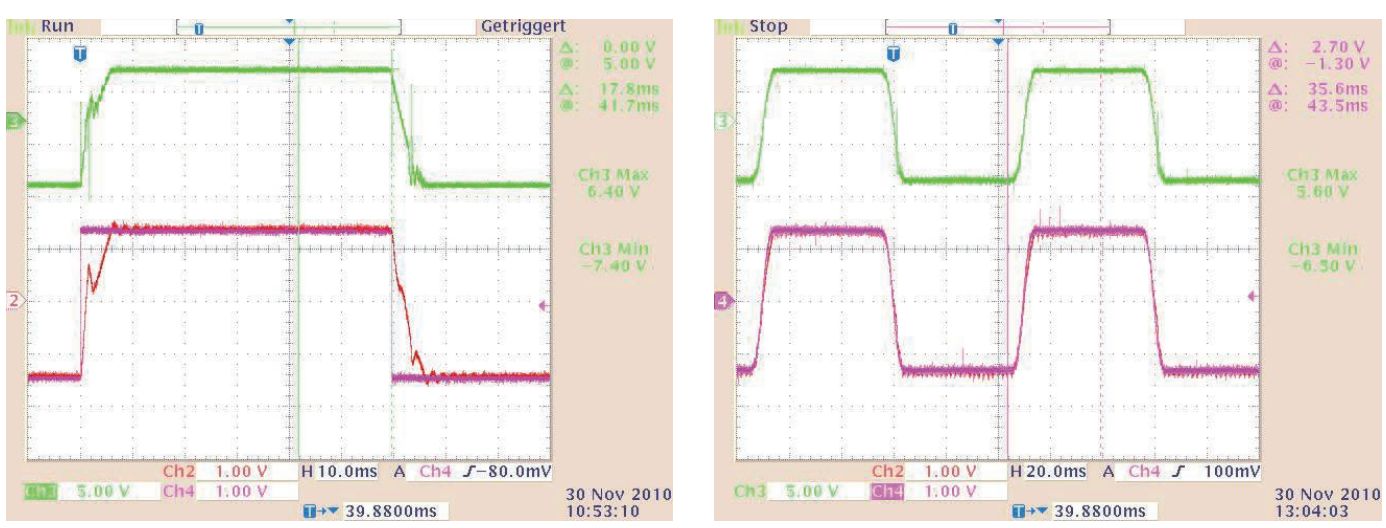

Fig. 10. Left: This diagram shows the achieved step response of the mirror when inserting it into the PID control loop. The speed of reaching the maximum deflection could be increased so that the mirror now just requires 8 ms anymore to reach a quasi stationary position. Right: Measurement when using the control software of mirrorcle, where the rectangular control signal is filtered by a Bessel low-pass with a bandwidth of about $180 \mathrm{~Hz}$.

For a scanner application the movement of the mirror should be a constant linear variation. Therefore the input signal was changed from a rectengular waveform to a triangular one. Fig. 11 illustrates the measurements results with this triactory.
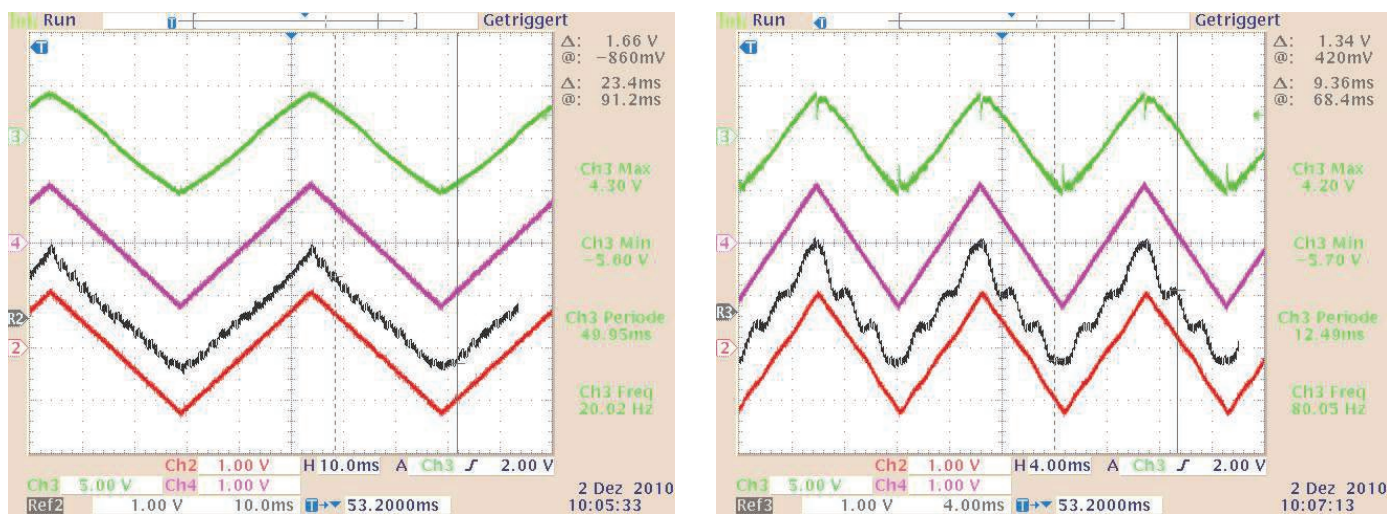

Fig. 11 Left: Signal 4 is the input signal, which sets the position of the mirror. Signal 3 represents the output of the PID circuit that controls the HV stage. Signal 2 indicates the mirror movement measured by the PSD. R2 is the signal of the PSD when driving the mirror in open loop. The measurement was done at a frequency of $20 \mathrm{~Hz}$. Right: The signals are still the same, but the measurements were done with a frequency of $80 \mathrm{~Hz}$. 
At higher frequencies it can be seen, that the resonance frequency of the mirror leaks into the measured position signal, if driving the mirror element in open loop. The reason is that every abrupt change in the control signal leads to an excitation of the eigenfrequency of the mirror. The effect is stronger if the frequency gets closer to its eigenfrequency. This means that it is very important to use a controlled driver design to achieve higher scanning frequencies and also much more precision.

\section{CONCLUSIONS}

In this article we presented a novel unit for closed-loop control of electrostatically driven resonant and quasistatic MOEMS mirrors, which will significantly improve the performance of these components. The first experimental results prove the possibility of driving the resonant mirror in a PLL system, but show that the phase jitter of such loop, although it is quite small still prohibits an accurate control near the resonance frequency of the mirror. Because of the narrow bandwidth of these mirrors close to their resonance this analog approach is not feasible.

The second experimental results prove the possibility to drive quasistatic mirror elements within a PID control loop. The control circuit leads to a 10 times faster position reaching than just driving the mirror in an open loop circuit and even much more precision. When using a PID controller it is also possible to achieve higher control frequencies. As long as the set-point signal's frequency is 10 times lower than the mirror's eigenfrequency the given trajectory is followed perfectly.

Both control concepts are perfectly convenient to synchronize more mirror elements, because after connecting them in parallel, every mirror controller locks onto the same input frequency or analog signal. We expect it to provide significant impact for applications of these MOEMS mirrors e.g. in compact projection devices.

\section{ACKNOWLEDGEMENTS}

The cooperation with all the scientific and industrial partners involved in the $7^{\text {th }}$ framework project "TACO" is gratefully acknowledged.

\section{REFERENCES}

[1] Kiang, M. H. , Solgaard, O., Muller, R. S., Lau, K. Y., "Micromachined polysilicon microscanners for barcode readers," IEEE Photon. Technol. Lett. 8, 1707-9 (1996)

[2] Zimmer, F., Grueger, H., Heberer, A., Wolter, A., Schenk, H., "Development of a NIR micro spectrometer based on a MOEMS scanning grating,", Proc. SPIE, 5455, 9 (2004)

[3] Schenk, H., Dürr, P., Kunze, D., Kück, H., "A new driving principle for micromechanical torsional actuators," Proc. of International Mechanical Engineering Congress and Exposition Nashville, 1, 333338 (1999)

[4] Tortschanoff, A., Lenzhofer, M., Frank, A., Wildenhain, M., Sandner, T., Schenk, H., Kenda, A., "Optical Position Feedback and Phase Control of MOEMS-Scanner Mirrors," Proc. SPIE, 7594 (2010)

[5] Milanovic, V., „Multilevel-Beam SOI-MEMS Fabrication and Application,“ IEEE/ASME Journal of Micromechanical Systems," Vol. 13, No. 1, 19-30, February, (2004)

Corresponding author: Martin Lenzhofer (martin.lenzhofer@ctr.at)

CTR Carinthian Tech Research AG,

Europastrasse 4/1, 9524 Villach, Austria,

phone: $+43 / 4242 / 56300226$, fax: $+43 / 4242 / 56300400$ 